\title{
Artificial Neural Network Based Fault Diagnosis of a Pulley-Belt Rotating System
}

\author{
Alaa Abdulhady Jaber ${ }^{\# 1}$, Khalid Mohsin $\mathrm{Ali}^{\# 2}$ \\ ${ }^{*}$ Mechanical Engineering Departement, University of technology, Iraq \\ E-mail: ${ }^{I} 20039 @$ uotechnology.edu.iq; ${ }^{2}$ khalid2771991@gmail.com
}

\begin{abstract}
Rotating machines are widely used in various industrial fields. Hence, an unexpected stoppage due to, for example, bad operating conditions or manufacturing error, has safety implications along with economic considerations. In this research, a fault detection system for a pulley-belt rotating system is developed and then different faults simulated in a test rig are investigated. Vibration signal monitoring is utilized since it represents a reliable approach for fault recognition in rotating machinery. Timedomain signal analysis technique is applied to extract some indicative features, such as root mean square, kurtosis and skewness. An artificial neural network (ANN) model is developed to detect the simulated faults. However, in addition to the machine healthy condition five fault types, such as unbalance in the driving pulley, wear in the belt and pulleys misalignment, have been simulated in the test rig. Two MEMS accelerometers (ADXL335), interfaced to Arduino MEGA 2560 as a data acquisition device, are used for vibration amplitude measurement. LabVIEW, which is a graphical programming software, is utilized to develop a signal capturing, analysis and feature extraction system. The result showed the effectiveness of the developed system in detection of different fault types in the pulley-belt system.
\end{abstract}

Keywords - fault diagnosis; artificial neural network; pulley-belt system; LabVIEW; arduino.

\section{INTRODUCTION}

Rotating machine faults diagnosis is typically based on vibration, acoustic emission, and motor signal analysis techniques. The condition monitoring process, however, has been taking considerable attention in recent years, due to the evolution of computer technologies and signal processing methods. Artificial intelligence techniques with their remarkable ability to derive meaningful information from complicated or imprecise data have recently been widely employed to extract patterns and detect trends that are too complex to be noticed by either human or other signal analysis techniques. A trained artificial system could be thought of as an expert system that can categories the given information. Artificial neural network (ANN), which represents an important type of artificial intelligence algorithms, is extensively applied for fault detection and diagnosis in different mechanical and electrical machines [1]-[3]. Jiang et al. [4] presented a fault recognition method for rotating machinery based on information entropy and a probabilistic neural network. The method first uses information entropy theory to extract three kinds of entropies from the vibration signals, namely, singular spectrum entropy, power spectrum entropy, and approximate entropy. The got experimental results verified the high performance of the proposed approach. Another paper has deliberated a belt drive system health monitoring considering vibration signal analysis [5]. An experimental test rig has been designed and fabricated and different faults types, such as side-cut-out and side-cut-in, in the belt have been simulated. For signal, acquiring NI DAQ system with LabVIEW software was utilized. From the results, the researchers have concluded that the belt fault nature has a significant effect on the characteristic frequency at oneX running speed for both driver and the driven shaft. Also, Li et al. [6] have considered conducting online belt conveyers monitoring by analyzing their vibration signals. Wavelet packet decomposition (WPD) has been used for signal analysis and features extraction and for features categorization, the support vector machine (SVM) was utilized. The advantage of this approach is that the fault location has easily and accurately been identified with a minimum number (one accelerometer) of sensors.

In term of the extracted features from vibration signals, research has conducted work for bearing fault detection in a rotating system based on time domain features analysis, such as root mean square and kurtosis, and artificial neural network for feature classification [7]. The developed model was then evaluated utilizing vibration data recorded from an induction motor subjected to some single point defects. The results showed that the proposed model is capable of fast 
processing identification of bearing faults. Another study has also carried on bearing fault diagnosis, but this time using acoustic emission signal analysis using time domain signal analysis along with fast Fourier transform (FFT) and envelope detection [8]. For envelope detection two approaches have been adopted which are Hilbert transform and order analysis. The results showed the high performance of the proposed algorithm in determining the presence and severity of fault and its location. Reddy and Sekhar [9] employed the ANN for unbalance and looseness identification in rotor-bearing systems via vibration signal monitoring. Two types of features, time domain, and frequency domain were extracted from the vibration signals and used for training and testing the ANN. Different unbalance levels were simulated in the experimental test rig. The researchers were observed that time domain features are giving better results over frequency domain features.

From the reviewed literature have noticed that the conducted researches in the area of belts health monitoring are quite a few. Thus, in this paper, an intelligent condition monitoring algorithm based on time-domain vibration signal analysis and ANN for feature classification is proposed for health monitoring of a pulley-belt rotating system, as discussed in the following sections.

\section{MAterial AND MEthod}

The used experimental test rig was previously designed by Hassan and Ali [10]. Square steel tubes having dimensions $3 \times 3 \mathrm{~cm}$ with $2 \mathrm{~mm}$ thickness were used to design the mechanical system structure. Different manufacturing processes, such as cutting, drilling, and welding, were followed in the design and installation steps. The final structure mass after assembly is $120 \mathrm{~kg}$. The steel structure was then filled with sand in order to make it more stable when it is in operation. Two steel pulleys are having $25 \mathrm{~cm}$ diameter were then utilized; one of them was used as a driving pulley and the other as driven. To carry the driving and driven shafts two stainless steel ball bearings were fixed on the designed structure. The flat, leather belt was utilized to transfer the motion between the drive and driven shafts. To generate the rotating motion a variable speed, Treadmill DC motor with speed control device has been employed. The motor speed can be varied from $250 \mathrm{rpm}$ to $3800 \mathrm{rpm}$ and capable of enduring load up to $100 \mathrm{~kg}$ with very little noise and vibration. For speed measurement, a digital tachometer type DT-2234A, which has a measuring range from $2.5 \mathrm{rpm}$ to $99999 \mathrm{rpm}$, was utilized. Vibration signal acquiring is done using two ADXL335 accelerometers, connected to Arduino MEGA 2560 microcontroller. Fig. 1 shows the final setup of the used test rig. However, the following subsections discuss the main conducted steps to get this research done.

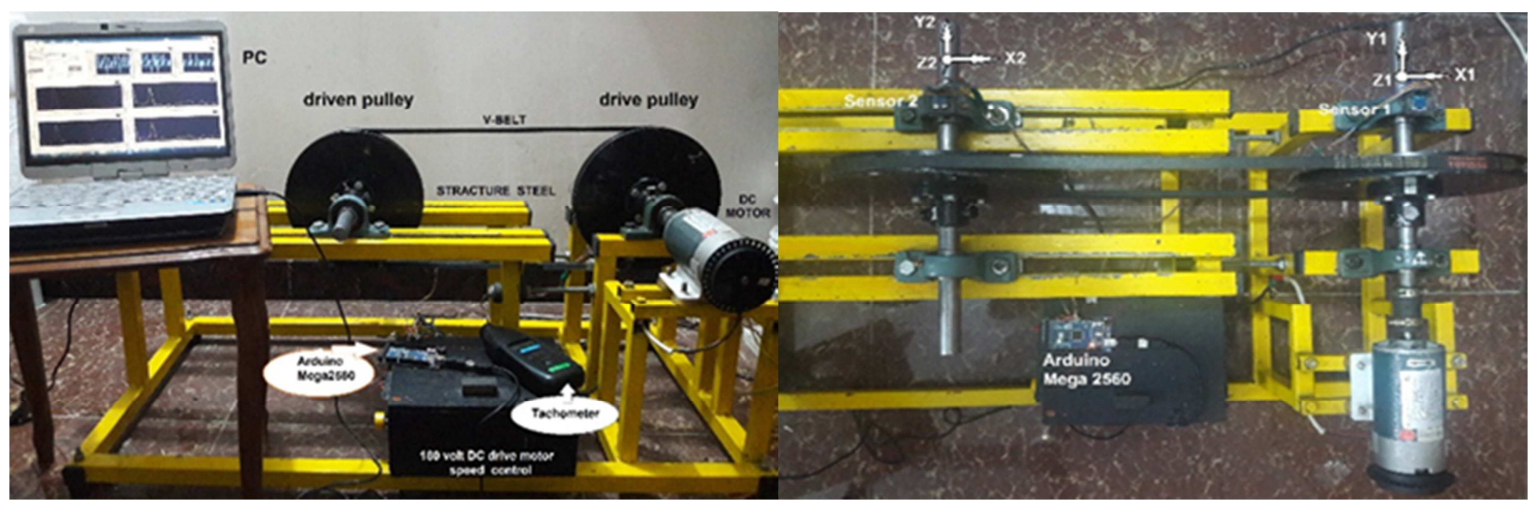

a) Front view

Fig.1 The used pulley-belt rotating system.

b) Top view

\section{A. Data Acquisition System Design}

As mentioned in the previous section the vibration signal is measured using two low-cost, triaxle vibration sensors, type ADXL335. This sensor is developed for wide applications and based on micro-electromechanical system (MEMS) technique. Due to the manufacturing technique of this sensor, it is less sensitive to temperature than other MEMS type of sensors, which is important since the temperature is increased when faults introduced in machines [11]. Arduino is an open source, low-cost platform based on inexpensive microcontroller board and user-friendly integrated development environment. In this research, Arduino MEGA 2560, which is based on the ATmega 2560 microcontroller and runs at $16 \mathrm{MHz}$ clock speed, is used. However, there are some advanced Arduino boards available, but the MEGA board was found to be enough for vibration signal acquisition in this research. It has 54 digital input/output pins (of which 15 pins can be used as PWM outputs), 15 analog inputs connected to a 10-bit ADC, 4 serial ports, a USB connection, a power jack, an ICSP (In circuit serial programming) header, and a reset button. To power the board up, it can simply be connected to a computer via a USB cable or using an AC-to-DC adapter or a battery to get started. However, the used accelerometers need between $1.8 \mathrm{~V}$ to $3.6 \mathrm{~V}$ to operate so the $3 \mathrm{~V}$ output on the Arduino have been used.

Programming Arduino is generally achieved via a textbased environment. National instrument (NI), however, has opened the door to Arduino users to program it graphically through LabVIEW. LabVIEW is software by which the user is relying on icons, instead of lines of text, to create different applications. Because of this, LabVIEW is called a graphical programming language. In LabVIEW there is a user 
interface, which is known as the front panel, can be constructed utilizing a set of tools and objects. There is another panel that contains the code that controls the front panel objects; it is referred to as the block diagram, which, in some ways, resembles a flowchart. LabVIEW programs are generally named virtual instruments (VIs) since their appearance and operation emulate the physical instruments, such as oscilloscopes and multimeters. LabVIEW Interface for Arduino (LIFA) is an extension of LabVIEW that allows researchers to communicate and control Arduino compatible cards from LabVIEW. After downloading LIFA from the Internet and installing it all the tools required using Arduino functions with LabVIEW will be available. Fig. 2 below shows the developed data acquisition software.

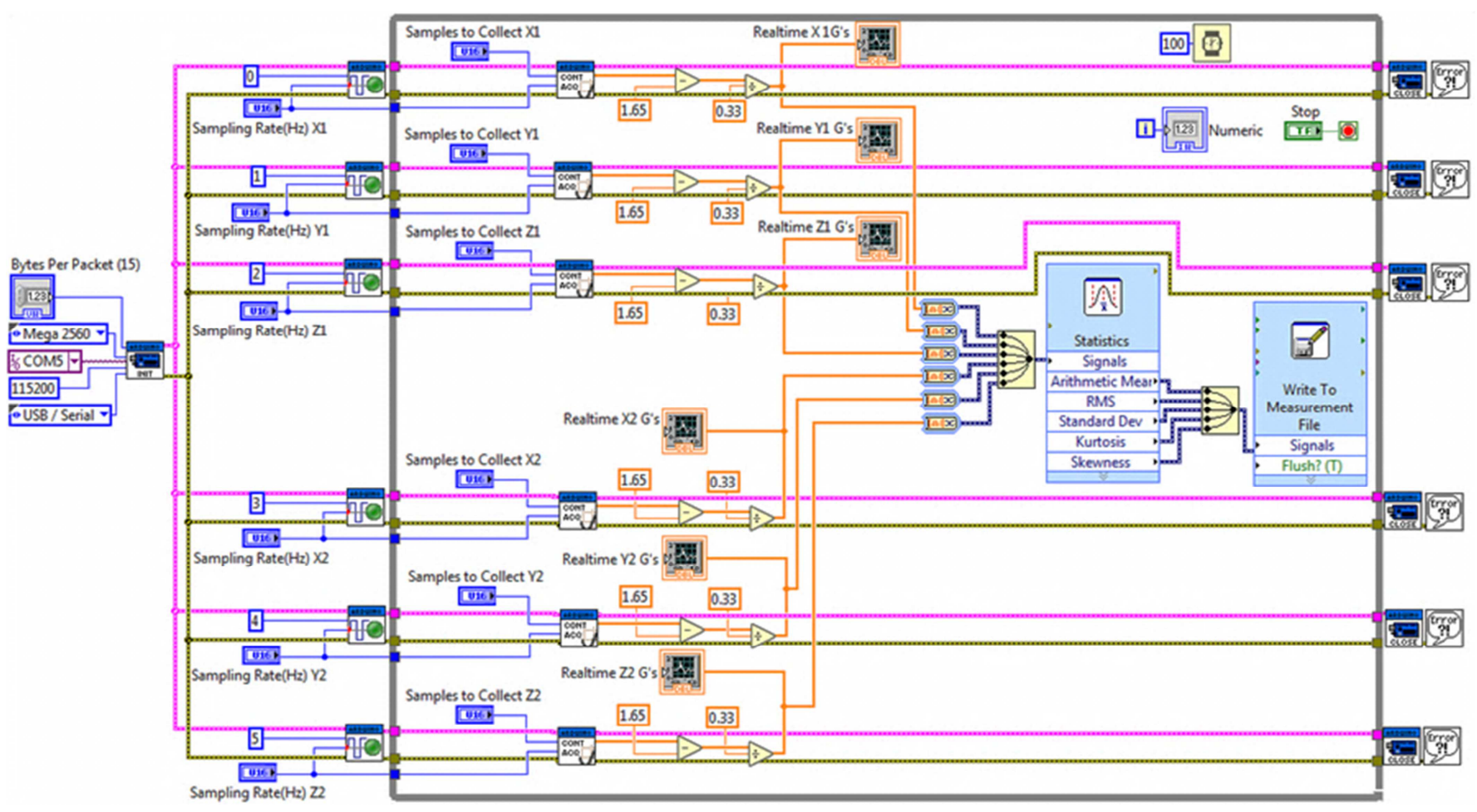

Fig. 2. The designed data acquisition software based on Arduino and LabVIEW.
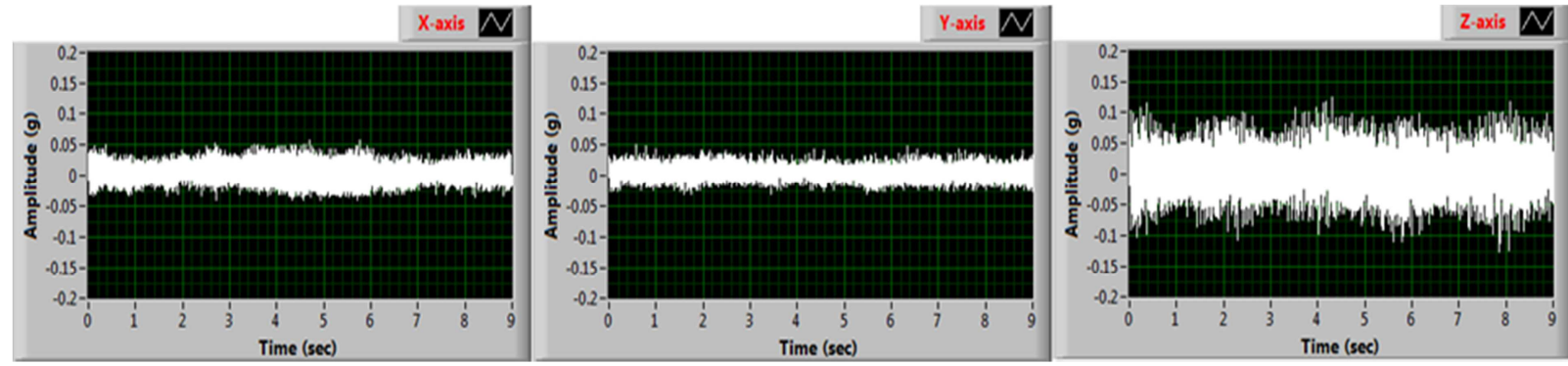

a) Healthy system
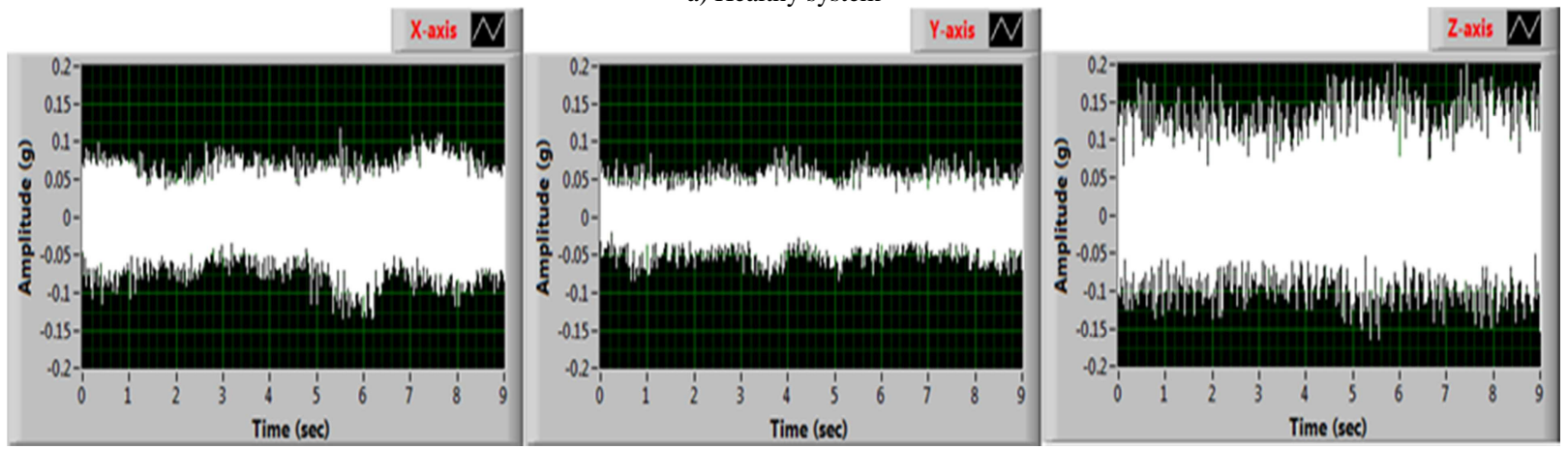

b) Faulty system

Fig. 3. Raw vibration signal captured from the pulley-belt system. 
Fig. 3 above shows raw vibration signals from the first accelerometer, which is on the drive shaft, when the system is healthy (Fig. 3a) and when a fault is introduced on the drive pulley (Fig. 3b), as discussed later. It can be noticed that there is a general increase in vibration amplitudes in the three axes when the fault is there. However, the increase is quite significant in $\mathrm{Z}$-axis, which represents the radial direction in the used accelerometer configuration in this research.

\section{B. Time Domain Signal Analysis}

Generally, signals carry information and features about the monitored system. Extracted features from the signal can be constant over some time or vary with time. Based on the features mentioned above' nature, signals can be classified as stationary or nonstationary. Signals whose statistical features do not change with time are known as stationary signals, which in turn are classified into deterministic and nondeterministic. Deterministic signals have specific distinct frequency components; signals emitted from rotating machines operating at a constant rotational speed are an example of stationary deterministic signals. Nondeterministic signals are well categorized by their statistical features, such as standard deviation, mean, variance, and so forth. On the other hand, signals that are having time domain statistical features changing with time are defined as nonstationary signals. The time domain statistical features of nonstationary signals are changing with time. The most common nonstationary signal is a transient type, such as the emitted signals from a defected mechanical part in a rotating system.

Captured signals have to be correctly analyzed in order to extract meaningful information from them. Stationary signals, however, can be analyzed using time domain or frequency domain signal analysis techniques [12]. Features extracted using time domain analysis give an overall impression of the monitored system. In frequency domain analysis, the frequency of occurrence of a mechanical event can be easily detected and related to the dynamics of the process. In this research, however, five time-domain features have been extracted from the captured signals, since time domain signal analysis represent a direct signal processing approach with simple calculations and each of the five parameters can reveal different characteristics about the monitored system [13]. The features are defined as follow [14]:

Mean ( $\boldsymbol{\mu})$ : This parameter measures the central tendency of the time domain signal and is given by

$$
\mu=\frac{1}{N} \sum_{i=1}^{N} x_{i}
$$

Root mean square (RMS): This parameter measures the energy level of the time domain signal and is given by

$$
R M S=\sqrt{\frac{1}{N} \sum_{i=1}^{N} x_{i}^{2}}
$$

Standard deviation $(\sigma)$ : This parameter measures the signal desperation about the mean and is given by

$$
\sigma=\sqrt{\frac{1}{N} \sum_{i=1}^{N}\left(x_{i}-\bar{x}\right)^{2}}
$$

Kurtosis (Ku): This parameter is statistically defined as the normalized fourth-order moment of the time domain signal, it is used to indicate the peak value of the probability density function (PDF) [15] and is given by

$$
K u=\frac{\sum_{i=1}^{N}\left(x_{i}-\bar{x}\right)^{4}}{(N-1) \sigma^{4}}
$$

Skewness (Sk): This parameter is known as the third centered moment of the time domain signal, it contains information about the symmetry of the signal distribution [15] and is given by

$$
S k=\frac{\sum_{i=1}^{N}\left(x_{i}-\bar{x}\right)^{3}}{(N-1) \sigma^{3}}
$$

Where $x_{i}$ a vector of the signal samples is, $\bar{x}$ is the mean of the collected samples and $N$ is the number of samples, respectively.

\section{Artificial Neural Network (ANN)}

The ANN is composed of some connected nodes termed artificial neurons, which are considered a simple form of biological neurons that are found in the brain. Each connection mimics the synapse in the biological systems where the signals can be easily transmitted from one connection to another in the ANN. The received signals by the artificial neurons are first treated and then utilized to signalize the artificial neurons that are linked to them. In common applications of the ANN, the signal at the connection between the artificial neurons are a real number, and the output of each artificial neuron is computed using a non-linear function of the summation of its inputs. The connections between neurons are named edges. Artificial neurons and edges usually have weights that adjusted as learning progresses. The weights either strengthen or weaken the signal at the connection. However, a threshold could be used with the artificial neurons such that only if the result of inputs addition passes that threshold the signal is sent to the following neurons.

Normally, neurons in the ANN are arranged in layers. Different layers may accomplish different types of transformations on their inputs. Signals are traveling from the first (input) layer to the last (output) layer, possibly after traversing the layers multiple times, during which the initial weights are corrected for output error minimization based on the backpropagation process (Fig. 4). The main objective of the ANN algorithm was to solve problems in the same approach that a human brain would. However, over time, attention focused on matching specific tasks, leading to deviations from biological concept. ANNs have been applied for a variety of tasks, including computer vision, voice/speech recognition, machine translation, social network filtering, video games, medical diagnosis and, of course, machines health monitoring. 


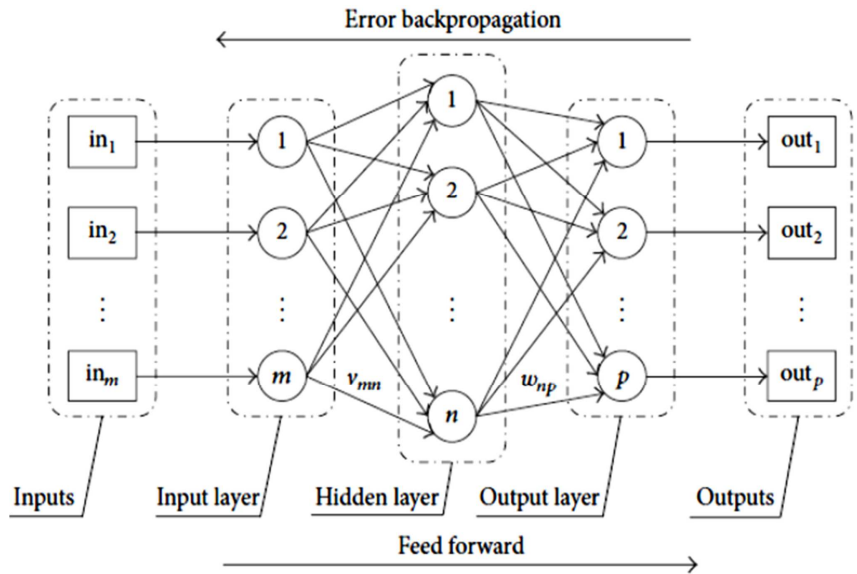

Fig. 4. Multi layers ANN with backpropagation [16].

\section{Design of the Artificial Neural Network}

Number of parameters have to be considered when structuring/designing any ANN model, such as number of hidden layers, number of neurons in these layers and type of activation functions that are used in the input, output and hidden layers. Matlab neural network toolbox has been utilized in this step. The neurons in the input layer are the same number as the input features to the network, which are ten extracted time domain features (five features from each sensor) computed from the measured vibration signal that have been previously illustrated. The features are proposed to be calculated by taking the resultant of each individual feature extracted from a signal axis, as shown in the following equations. Thus, the total extracted features are ten (five features from each accelerometer).

$$
\begin{gathered}
\mu_{\text {resultant }}=\mu_{\mathrm{X}}+\mu_{\mathrm{Y}}+\mu_{\mathrm{Z}} \\
\mathrm{RMS}_{\text {resultant }}=\mathrm{RMS}_{\mathrm{X}}+\mathrm{RMS}_{\mathrm{Y}}+\mathrm{RMS}_{\mathrm{Z}} \\
\sigma_{\text {resultant }}=\sigma_{\mathrm{X}}+\sigma_{\mathrm{Y}}+\sigma_{\mathrm{Z}} \\
\mathrm{Ku}_{\text {resultant }}=\mathrm{Ku}_{\mathrm{X}}+\mathrm{Ku}_{\mathrm{Y}}+\mathrm{Ku}_{\mathrm{Z}} \\
\mathrm{Sk}_{\text {resultant }}=\mathrm{Sk}_{\mathrm{X}}+\mathrm{Sk}_{\mathrm{Y}}+\mathrm{Sk}_{\mathrm{Z}}
\end{gathered}
$$

The neurons in the output layer are six while the hidden layers' neurons are 45 and 25, respectively. A log-sigmoid transfer function is selected for both hidden layers and linear function for the output layer. However, the ANN has been trained using different algorithms, for instance, gradient decent (traingd), scaled conjugate gradient algorithm (trainscg), resilient back propagation algorithm (trainrp) and Levenberg-Marquardt algorithm (trainlm), etc. and the latter was found the best.

Other parameters, such as a number of training iterations and the learning rate and even the number of neurons in the hidden layers, have to be carefully tuned in the designing process. These parameters, though, are generally adjusted based on a trial-and-error procedure. Hence, the training process is terminated if one of the following conditions occurs: the maximum number of epochs is reached 100; the performance is minimized to the goal of 10-5; the gradient goes below the minimum value of 10-7. In order to get the developed ANN model converged, the input data vector, which consists of six groups representing the health conditions of the pulley-belt system, has been normalized to be within the range of $[0,1]$ based on Equation (11) below [17].

$$
X_{s}=\frac{X-X_{\min }}{X_{\max }-X_{\min }}
$$

Where $X_{S}$ represent the normalized input variables, $X$ the input variables before normalization, $X_{\min }$ and $X_{\max }$ are the minimum and maximum values of the inputs, respectively. To define the health conditions of the designed system at the output layer a binary classification scheme is used. Thus, the result of the output layer has to be $\left(\begin{array}{llllll}1 & 0 & 0 & 0 & 0 & 0\end{array}\right)$ for the healthy condition, ( $\left(\begin{array}{llllll}0 & 1 & 0 & 0 & 0 & 0\end{array}\right)$ for drive pulley unbalance, $\left(\begin{array}{llllll}0 & 0 & 1 & 0 & 0 & 0\end{array}\right)$ for drive pulley damage, $\left(\begin{array}{llllll}0 & 0 & 0 & 1 & 0 & 0\end{array}\right)$ for belt neck, ( $\left(\begin{array}{llllll}0 & 0 & 0 & 0 & 1 & 0\end{array}\right)$ for belt worn and ( $\left(\begin{array}{llllll}0 & 0 & 0 & 0 & 0 & 1\end{array}\right)$ for misaligned pulleys. Fig. 5 below is showing the general steps that have been followed in designing the ANN in this research.

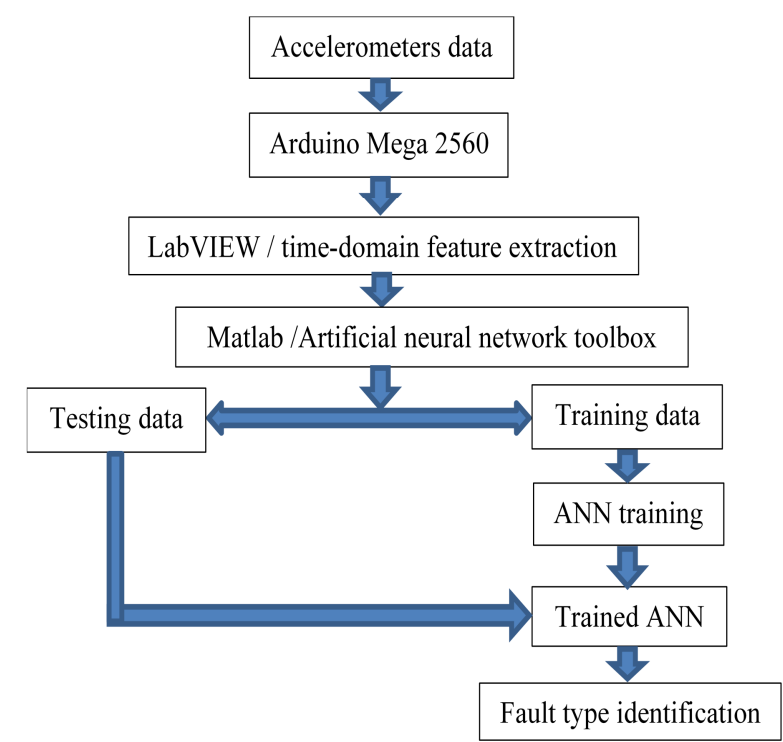

Fig. 5. Flowchart showing steps of designing the ANN.

\section{RESULTS AND DISCUSSION}

The developed system is firstly operated without fault with the motor rotating speed is $1500 \mathrm{rpm}$, initial belt tension is $30 \mathrm{~N}$ and the center distance is $50 \mathrm{~cm}$. Then, five different faults are created, as explained in the following. Unbalance fault is simulated by attaching small magnets (50 g) on the driving pulley (Fig. 6), this mass develops centrifugal force in the radial directions. A driving pulley fault is imitated by adding little bump inside the groove of the pulley. A side cut-out in the belt is introduced as another fault type. To simulate the belt slippage, due to wear in belt material, little amount of oil is added to the contact area between the pulley and belt. The last fault is a misalignment in the pulleys, which is made by moving one of the pulleys 
on its shaft. However, each time on the fault is simulated in the system and at that fault vibration signals from the two accelerometers are gathered and instantly analysed and features extracted using the developed LabVIEW code. The extracted features are then uploaded to Matlab for purpose of ANN design. The neural network toolbox in Matlab takes, by default, $70 \%$ of the data for training and $15 \%$ for testing and the left $15 \%$ of data is for validation.

Performance evaluation of the designed ANN is assessed using the mean square error (MSE), indicated in Fig. 7 and Table 1, and the correlation coefficient (R), which is presented in Fig. 8 where the ANN estimation and target values are highly overlapped. For more accurate validation of the developed ANN, unseen data have been presented to it. The unseen data consist of 1200-sets, each 200-set represent a health condition of the mechanical system and only one fault type is presented at a time. Fig. (9) shows the ANN classification results; as can be noticed that when the system is healthy the first neuron in the output layer is 1 and stay as it is for 200 data sets whereas the other neurons are zeroes. Then, the second neuron becomes 1 when the first fault is introduced and the other neurons are zeroes and so on for the other fault types. From the result in Fig. 8 it can be clearly seen that the faults are easily identified, thus, the designed ANN is feasible for the application in this research.

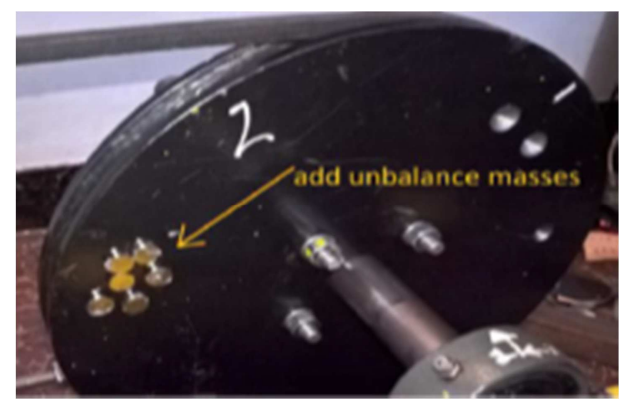

a) Pulley unbalance

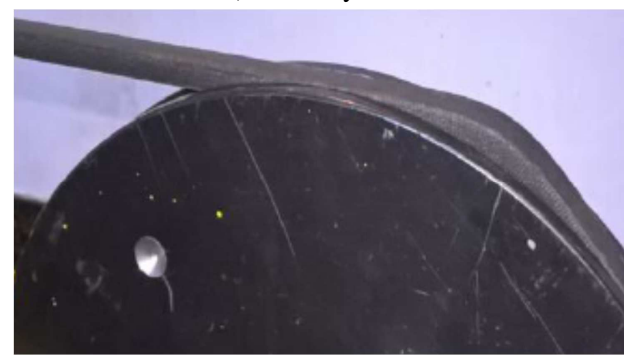

b) Little bump in the driving pulley

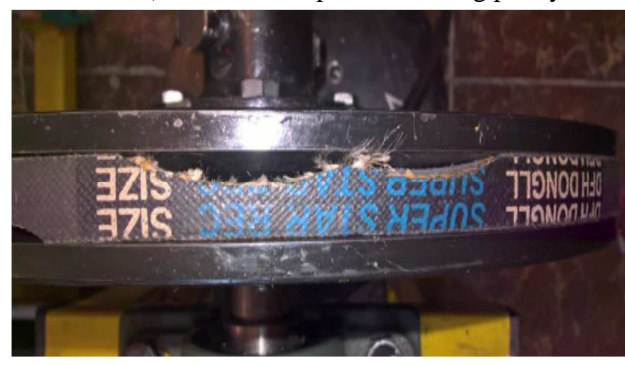

c) Belt side cut-out

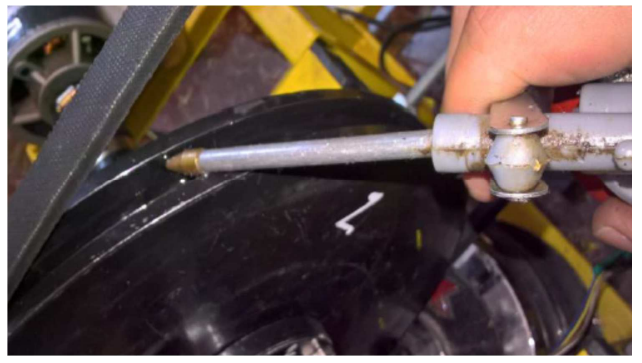

d) Slipping in the belt

Fig. 6. Number of simulated faults in the designed pulley-belt test rig.

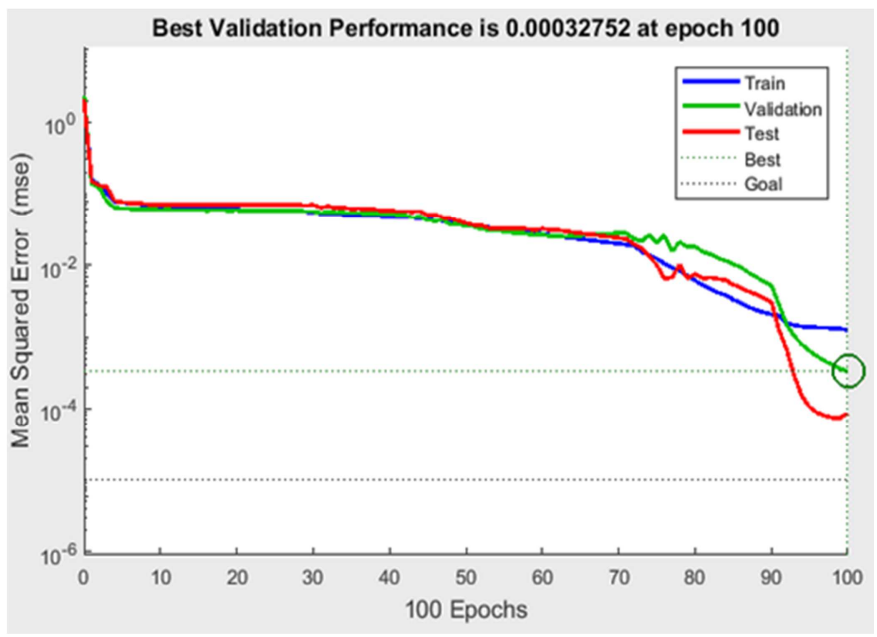

Fig. 7. The performance plot of the designed ANN.

TABLE I

PERFormanCE (MSE) OF THE DESIGNED ANN

\begin{tabular}{|l|l|}
\hline Train & 0.0013 \\
\hline Validation & 0.00032 \\
\hline Test & 0.000085 \\
\hline
\end{tabular}
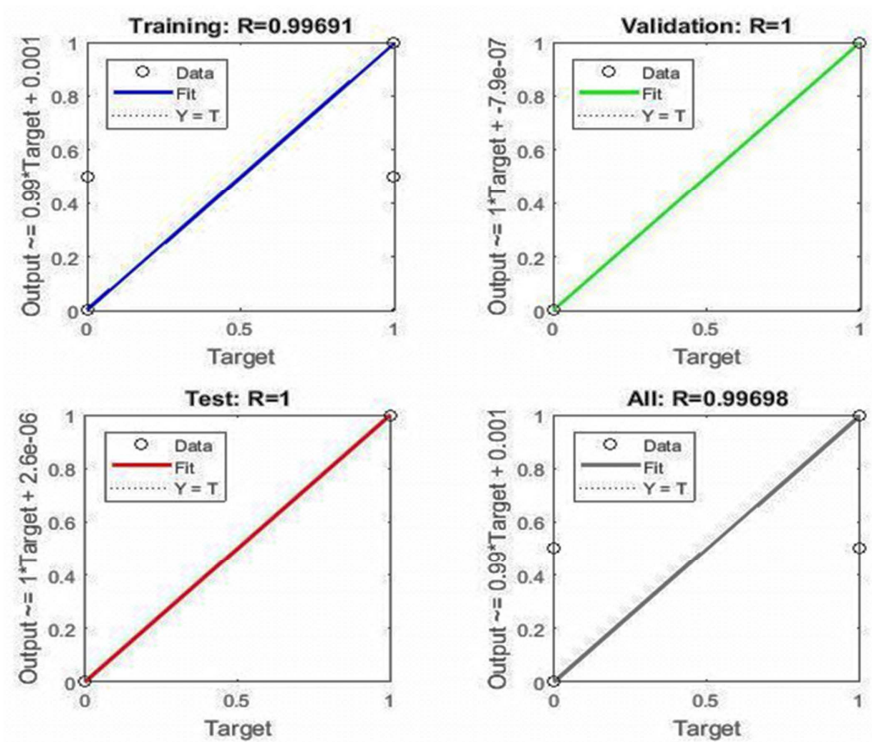

Fig. 8. Correlation coefficient of the developed ANN 


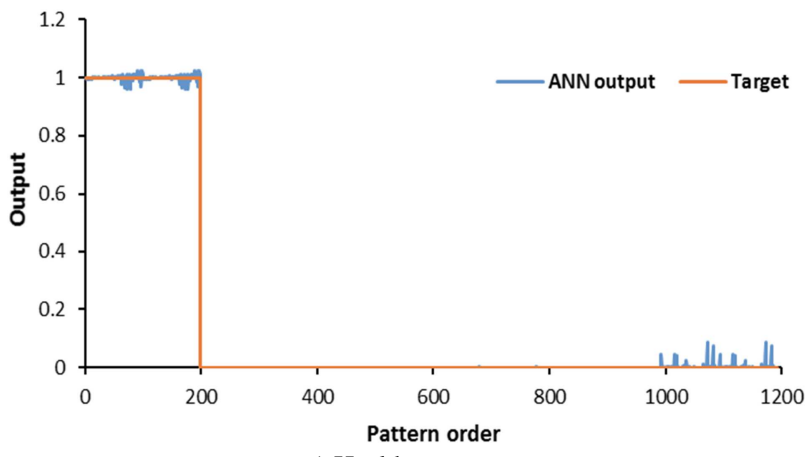

a) Healthy system.

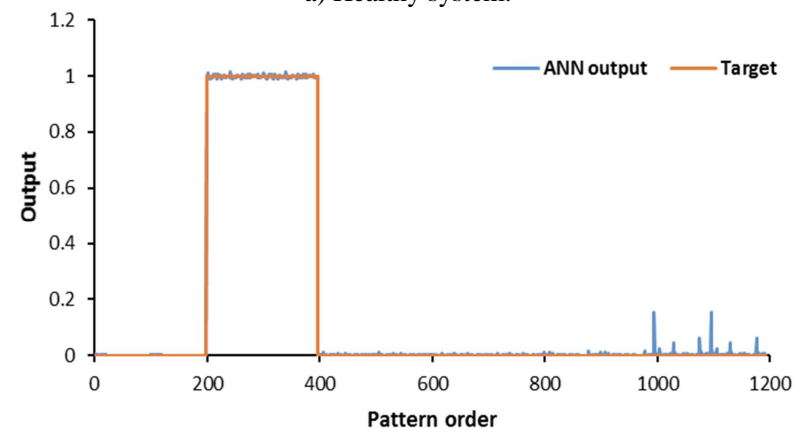

b) Unbalance fault.

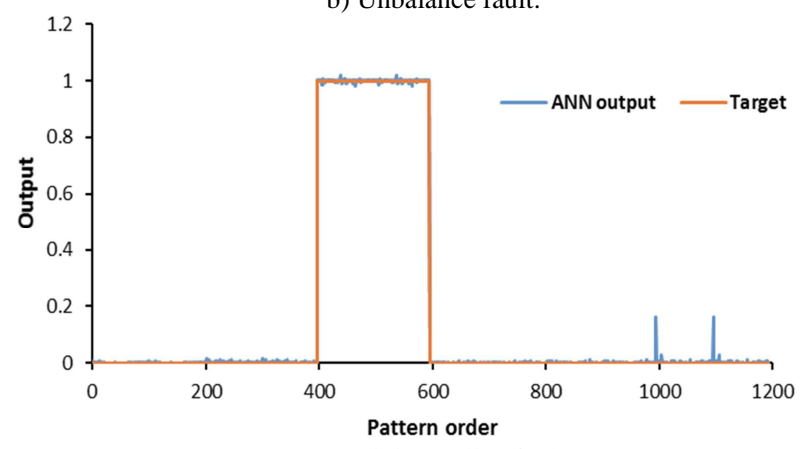

a) Driving pulley fault.

Fig. 9. Result of the designed ANN when unseen data are presented.
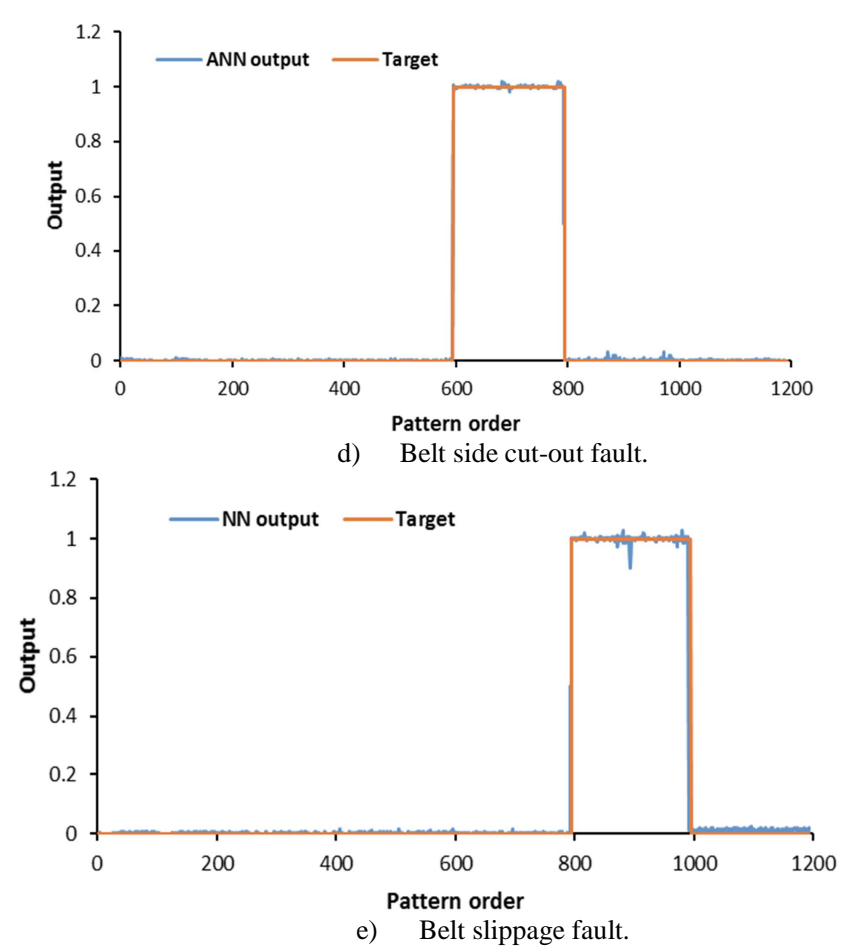

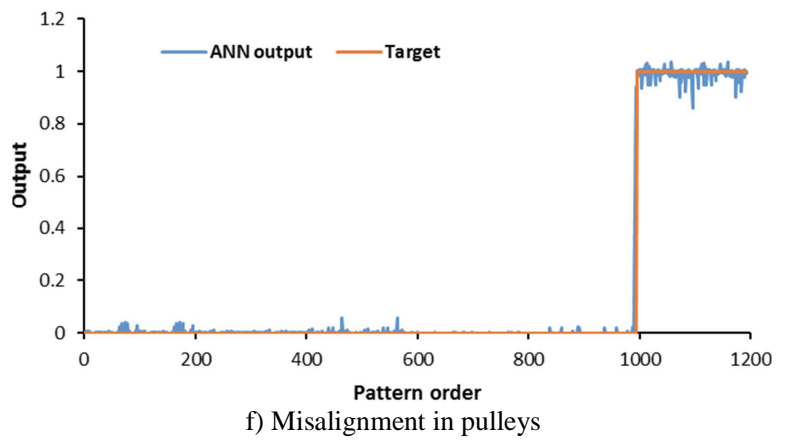

Fig. 9. Result of the designed ANN when unseen data are presented (continued).

\section{CONCLUSION}

In this research, a fault detection system for a pulley-belt rotating system has been developed based on backpropagation artificial neural network (ANN). Pulleybelt simulator, in which DC motor, flat belt and steel structure were used, was firstly designed and fabricated for this research. A low coast data acquisition system was constructed using two MEMS accelerometers, Arduino MEGA 2560 microcontroller and LabVIEW software. Extracted features play a vital role in any health monitoring system and especially in training a high performance ANN. Thus, time-domain signal analysis based feature extraction was conducted. Various features, such kurtosis and skewedness, were computed. Extensive experimental investigation was carried out on the pulley-belt simulator. Firstly, vibration signals were acquired, and then the features are extracted, from the system when it is healthy. More signals and further features were acquired when five different faults were introduced. The imitated faults were an unbalance in the driving pulley, a driving pulley fault, belt side cut-out and misalignment in the pulleys. It was observed that time-domain vibration signal analysis is giving good results in differentiation between the healthy and fault conditions. The same conclusion, however, was previously obtain by Reddy and Sekhar [9]. The current study can be extended to detect other fault categories, such as bearings faults, or multiple faults simultaneously.

For future work, further improvements are suggested to be made on the developed detection system. They are mainly based on adding wireless capability on the data acquisition system and also applying the ANN concurrently with feature extraction process using LabVIEW, in order to make a fully automated detection approach. Moreover, mobile application could also be constructed in order to allow the people who are in charge to monitor the machines remotely.

\section{REFERENCES}

[1] D. Ying, H. Yigang, and S. Yichuang, "Fault diagnosis of analog circuits with tolerances using artificial neural networks," in IEEE Asia-Pacific Conference on Circuits and Systems. Electronic Communication Systems. , 2000, pp. 292-295.

[2] H. Li, Y. Zhang, and H. Zheng, "Gear fault detection and diagnosis under speed-up condition based on order cepstrum and radial basis function neural network," Journal of Mechanical Science and Technology, vol. 23, pp. 2780-2789, 2009.

[3] D. Pandya, S. Upadhyay, and S. Harsha, "Ann based fault diagnosis of rolling element bearing using time-frequency domain feature," International Journal of Engineering Science and Technology, vol. 4, pp. 2878-2886, 2012. 
[4] Q. Jiang, Y. Shen, H. Li, and F. Xu, "New fault recognition method for rotary machinery based on information entropy and a probabilistic neural network," Sensors, vol. 18, 2018.

[5] A. A. A. Bulushi, G. R. Rameshkumar, and M. Lokesha, "Fault Diagnosis in Belts using Time and Frequency based Signal Processing Techniques," International Journal of Multidisciplinary Sciences and Engineering, vol. 6, 2015.

[6] W. Li, Z. Wang, Z. Zhu, G. Zhou, and G. Chen, "Design of Online Monitoring and Fault Diagnosis System for Belt Conveyors Based on Wavelet Packet Decomposition and Support Vector Machine," Advances in Mechanical Engineering, vol. 5, p. 797183, 2013.

[7] C. Wu, T. Chen, R. Jiang, L. Ning, and Z. Jiang, "ANN Based Multiclassification Using Various Signal Processing Techniques for Bearing Fault Diagnosis," International Journal of Control and Automation, vol. 8, pp. 113-124, 2015.

[8] A. R. Bhendea, G. K. Awarib, and S. P. Untawalec, "Comprehensive bearing condition monitoring algorithm for incipient fault detection using acoustic emission," Jurnal Tribologi, vol. 2, 2014.

[9] M. C. S. Reddy and A. S. Sekhar, "Application of Artificial Neural Networks for Identification of Unbalance and Looseness in Rotor Bearing Systems," International Journal of Applied Science and Engineering, vol. 1, pp. 69-84, 2013.

[10] A. R. Hassan and K. M. Ali, "Effects Of Rotational Speed, Center Distance And Diameter Ratios On The Dynamic Response Of
Pulley-Belt System Depends On Vibration Analysis," Al-Qadisiyah Journal For Engineering Sciences, vol. 10, 2017.

[11] V. K. Patel and M. N. Patel, "Development of Smart Sensing Unit for Vibration Measurement by Embedding Accelerometer with the Arduino Microcontroller," International Journal of Instrumentation Science, vol. 6, pp. 1 - 7, 2017.

[12] J. Yan, Machinery Prognostics and Prognosis Oriented Maintenance Management: John Wiley \& Sons, 2015.

[13] S. Fu, K. Liu, Y. Xu, and Y. Liu, "Rolling Bearing Diagnosing Method Based on Time Domain Analysis and Adaptive Fuzzy Means Clustering," Shock and Vibration, vol. 2016, p. 8, 2016.

[14] J. K. Sinha, Vibration Analysis,Instruments, and Signal Processing, First Edition ed.: CRC Press, 2014.

[15] W. Caesarendra and T. Tjahjowidodo, "A Review of Feature Extraction Methods in Vibration-Based Condition Monitoring and Its Application for Degradation Trend Estimation of Low-Speed Slew Bearing," Machines, vol. 5, p. 21, 2017.

[16] M. czkiewicz and T. Barszcz, "Application of Artificial Neural Network for Damage Detection in Planetary Gearbox of Wind Turbine," Shock and Vibration, vol. 2016, p. 12, 2016.

[17] M. W. Ahmad, M. Mourshed, and Y. Rezgui, "Trees vs Neurons Comparison between Random Forest and ANN for high-resolution prediction of building energy consumption," Energy and Buildings, vol. 147, 2017. 